\title{
Universalities in Halo Nuclei
}

\author{
M.T. Yamashita \\ Instituto de Física Teórica, UNESP - Univ Estadual Paulista, C.P. 70532-2, CEP 01156-970, São \\ Paulo, SP, Brazil
}

\begin{abstract}
In this contribution I provide an overview of our group papers involving universalities in light exotic nuclei. It is also made a connection of these systems with some weakly bound ultracold molecules.
\end{abstract}

Keywords: Few-body systems

PACS: $25.45 .-\mathrm{v}$

\section{INTRODUCTION}

In the few-body physics context the tiny line between bound and scattering states (distinguished, respectively, by a discrete and a continuous spectrum) is what we ${ }^{1}$ call threshold. Studies of systems in the vicinity of a threshold can reveal very interesting structures. A curious result related to these weakly-bound structures is called universality and the physical observables linked to this are called universal. More specifically, physical observables are called universal if they do not depend on the details of the short range interaction. Thus, we can say that some systems that are different at short distances can present identical behavior at long [1].

There is a class of nuclei close to the limit of stability that is called halo nuclei (or exotic nuclei) [2]. The halo structure for a nucleus close to the neutron dripline is usually composed by a core and one or two loosely bound neutrons. The first experimental observation of a halo nucleus $\left({ }^{6} \mathrm{He}\right)$ was made in the 30's [3], but the "modern" studies of these nuclei began only in 1985 with the observation of a huge increase of ${ }^{11} \mathrm{Li}$ cross section in an experiment that was measuring the cross section of Li isotopes colliding with some targets [4]. That increase was first interpreted as due to the ${ }^{11} \mathrm{Li}$ structure that could be formed by a core, ${ }^{9} \mathrm{Li}$, and two weakly-bound neutrons. This assumption was experimentally proved in 1989 [5].

\section{EFIMOV STATES}

One remarkable phenomenon involving universal features is the appearance of Efimov states [6]. An infinite number of these three-body bound states appears when the energies of the two-body subsystems go to zero. Efimov states were predicted theoretically in

\footnotetext{
${ }^{1}$ I used "we" when referring to our group - formed by A. Delfino, T. Frederico and L. Tomio - or few-body physics community.
}

CP1245, The XXXII Brazilian Workshop on Nuclear Physics 
the nuclear context in 70's but their first experimental evidence was made in ultracold atomic traps only in 2006 [7]. A peculiar characteristic of Efimov states is the ratio between two consecutive three-body binding energies, $E_{3}$. When the energy of the twobody subsystems, $E_{2}$, tends to zero this ratio tends to

$$
E_{3}^{(n)} / E_{3}^{(n+1)} \rightarrow 515.03
$$

with $n \rightarrow \infty$. The size of these systems also obeys a ratio of $\approx 22.7$, i.e., the state $(n+1)$ is $\approx 22.7$ times greater than state $n$ (these ratios are valid for three identical bosons with equal masses).

The appearance of Efimov states is closely related to the possibility of collapse of the three-body systems (Thomas collapse) [8]. Rescaling the momentum variables and two- and three-body binding energies as $\vec{x}=\frac{\vec{p}}{\mu_{(3)}}, \vec{y}=\frac{\vec{q}}{\mu_{(3)}}, \varepsilon_{2}=\frac{E_{2}}{\mu_{(3)}^{2}}, \varepsilon_{3}=\frac{E_{3}}{\mu_{(3)}^{2}}$, where $\mu_{(3)}^{2}$ is the three-body subtraction energy (equivalent to an energy momentum cutoff), we can write the three-body bound state integral equation for a Dirac- $\delta$ potential using the dimensionless variables as ( $\hbar=m=1$, where $m$ is the boson's mass)

$$
\chi(\vec{y})=\frac{-\pi^{-2}}{ \pm \sqrt{\varepsilon_{2}}-\sqrt{\varepsilon_{3}+\frac{3}{4} y^{2}}} \int d^{3} x\left[G\left(\vec{x}, \vec{y} ; \varepsilon_{3}\right)-G\left(\vec{x}, \vec{y} ; \mu_{(3)}^{2}\right)\right] \chi(\vec{x}),
$$

with $\chi(\vec{x}) \equiv \mu_{(3)}^{3 / 2} \varphi(\vec{p})$, where $\varphi$ is the Faddeev component of the three-boson bound state vertex, for zero angular momentum. The Green's function is given by $G(\vec{x}, \vec{y} ; E)=$ $\left(E+y^{2}+x^{2}+\vec{y} \cdot \vec{x}\right)^{-1}$. The + and - signs in front of $\sqrt{\varepsilon_{2}}$ distinguish, respectively, a bound from a virtual state ${ }^{2}$. The details of the formalism can be found in Ref. [9].

The number of three-body bound states, given by the values of $\varepsilon_{3}$ satisfying Eq. (1), grows without limit when $\varepsilon_{2}$ decreases to zero. This limit $\left(\varepsilon_{2} \rightarrow 0\right)$ can be achieved either by $E_{2} \rightarrow 0$ (with a fixed $\mu_{(3)}$ ) or by $\mu_{(3)} \rightarrow \infty$ (with $E_{2}$ fixed). In this last case the system collapses: $E_{3}^{(0)}=\varepsilon_{3}^{(0)} \mu_{(3)}^{2} \rightarrow \infty$. This is known as the Thomas collapse of the three-body ground state. Therefore, the Thomas and the Efimov states are given by the same limit, $\varepsilon_{2} \rightarrow 0$, of Eq. (1), and are related only by a scale transformation. Fig. 1 shows the appearance of Efimov states when $\varepsilon_{2}$ is decreased.

The Thomas collapse is also related to the number of physical scales that are necessary to describe a system [10]. For a three-boson system interacting by an $s$-wave in three dimensions, as the Thomas collapse is present, we need a three-body scale beyond the two-body one to completely describe the observables in the universal regime, $a / r_{0} \gg 1$, in which the two-body scattering length, $a$, is much greater than the range of the potential, $r_{0}$. Translating this into an equation, we can write:

$$
\mathscr{O}\left(E, E_{2}, E_{3}\right)=\left(E_{3}\right)^{\eta} \mathscr{F}\left(\frac{E}{E_{3}}, \frac{E_{2}}{E_{3}}\right) .
$$

\footnotetext{
${ }^{2}$ A virtual state is a quasi-bound state in the second energy Riemann sheet.
} 


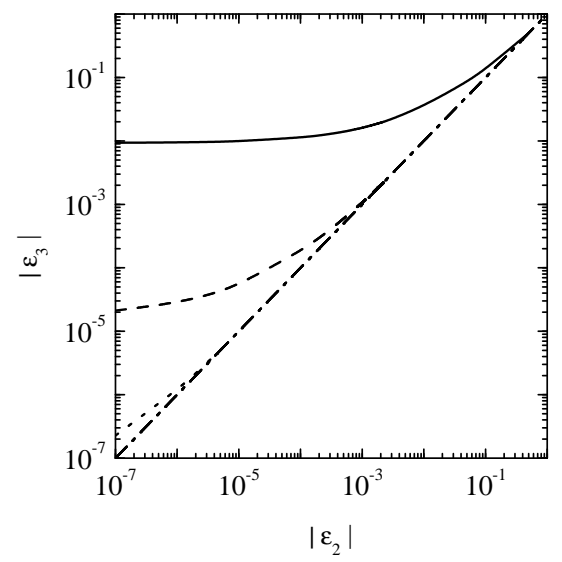

FIGURE 1. Appearance of Efimov states when $\varepsilon_{2}$ approaches zero. The solid, dashed and dotted lines represent, respectively, the ground state and the first and second excited states. The dash-dotted line is $\varepsilon_{2}=\varepsilon_{3}$. A similar figure can be found in Ref. [9].

Any three-body observable, $\mathscr{O}$, at an energy $E$ with dimension $\left(E_{3}\right)^{\eta}$ can be written as a scaling function dependent only on the two- and three-body scales, that can be chosen as their binding energies.

The above assumption brings us a natural question: if one more boson is added to the system should we also add one more scale, independent of the two- and three-body scales, to describe the four-boson system? This is still an open question that deserves investigation $[11,12]$.

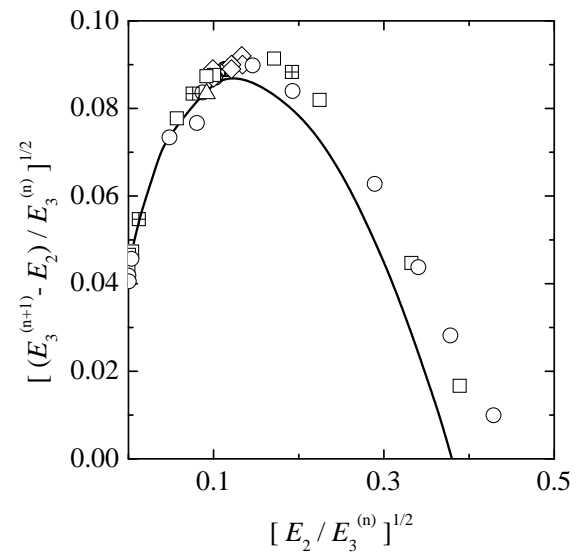

FIGURE 2. Trimer excited $(\mathrm{n}+1)^{\text {th }}$ state energy as a function of the ratio of the dimer energy and trimer $\mathrm{n}^{\text {th }}$ bound state energy. The symbols represent results from other realistic calculations (for references of the realistic data please see Ref. [9]). A similar figure can be found in Ref. [9].

Eq. (2) provides an interesting manner to verify if a system is universal or not. Fig. 2 shows a "scaling plot", where the energies were calculated using our zero-range 
approach. The zero-range calculation is the limit of short range potentials and is very convenient to study weakly-bound systems. The points are realistic calculations.

We can see for small two-body energies that all points match our curve. As we increase $E_{2}$ the difference between realistic and zero-range calculations also increases indicating that we are leaving the universal regime and we need more parameters to describe the system. These weakly-bound systems have a wave function that extends much beyond the range of the potential, thus another way to verify the universality is to check the probability of the wave function to reach the classical forbidden region [2].

\section{HALO NUCLEI}

In this section I will consider only three-body halo nuclei approached by an inert dimensionless core and two loosely bound neutrons. Using this approximation we can classify halo nuclei as indicated in Fig. 3 [13].

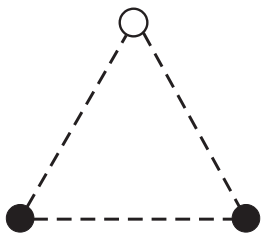

Borromean

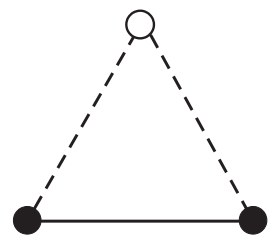

Tango

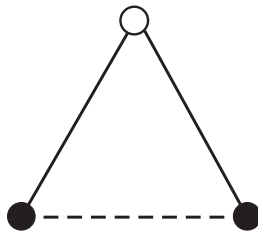

Samba

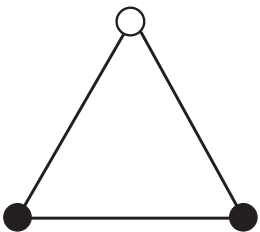

All-Bound

FIGURE 3. Classification scheme for a three-body $\alpha-\alpha-\beta$ system. A solid line represents a bound state and a dashed line an unbound (virtual) state. For two neutron halo nuclei $\alpha$ represents a neutron, in this case the subsystem $\alpha-\alpha$ is always unbound. Figure from Ref. [13].

The name "Borromean", representing a three-body bound system where all pairs are unbound, comes from the borromean rings, a structure where three rings are connected and the pairs are not. The name "Tango" was given because the bound pair should move in a very harmonic way to have a three-body bound system [14]. When two twobody subsystems are bound the three-body system can "shake" a little bit more than in the "Tango" type and still remaining bound, thus we decided to give it the name "Samba" [13].

If the above systems are in the universal regime we can systematically study interesting scaling plots in order to verify how universal are some observables. In this case the scaling function can be written as

$$
\mathscr{O}\left(E, E_{3}, E_{\mathrm{nn}}, E_{\mathrm{nA}}\right)=\left(E_{3}\right)^{\eta} \mathscr{F}\left(\frac{E}{E_{3}}, \frac{E_{\mathrm{nn}}}{E_{3}}, \frac{E_{\mathrm{nA}}}{E_{3}} ; \mathrm{M}\right),
$$

where $E_{\mathrm{nn}}$ and $E_{\mathrm{nA}}$ are, respectively, the energies of the two-body subsystems neutronneutron and neutron-core. $\mathrm{M}$ is the mass of the core $\mathrm{A}$.

Fig. 4 presents an example of scaling plot relating the root-mean-square radius and energies (for other scaling plots see Ref. [13]). In this plot we use the following definitions: $E_{\mathrm{nA}} / E_{3} \equiv K_{\mathrm{nA}}^{2}$ and $E_{\mathrm{nn}} / E_{3} \equiv K_{\mathrm{nn}}^{2}$ (for both bound or virtual state energies), such that

$$
K_{\mathrm{nA}}= \pm \sqrt{E_{\mathrm{nA}} / E_{3}} \text { and } K_{\mathrm{nn}}= \pm \sqrt{E_{\mathrm{nn}} / E_{3}} .
$$




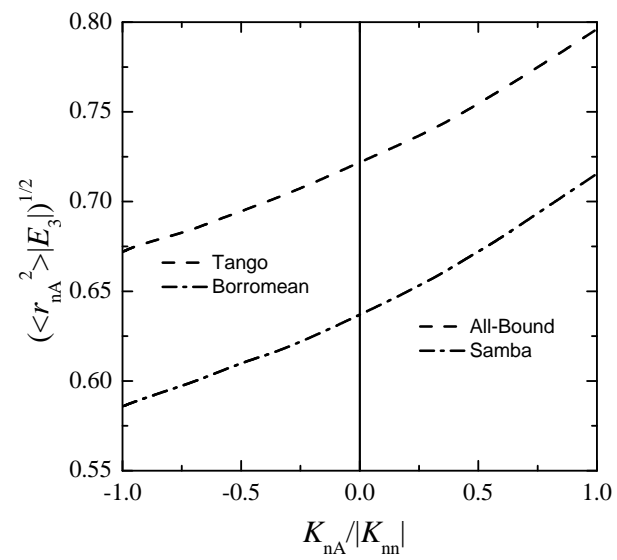

FIGURE 4. Dimensionless product ( $\hbar=m_{\mathrm{n}}=1$, where $m_{\mathrm{n}}$ is the neutron's mass) $\sqrt{\left\langle r_{\mathrm{nA}}^{2}\right\rangle\left|E_{3}\right|}$ for $\mathrm{M}=200$ and $E_{\mathrm{nn}} / E_{3}=0.1$ as a function of $K_{\mathrm{nA}} /\left|K_{\mathrm{nn}}\right|$. A bound nn pair is represented with dashed line; and a virtual $\mathrm{nn}$ pair with dot-dashed line. This figure shows the transitions Tango $\rightarrow$ All-Bound and Borromean $\rightarrow$ Samba. A similar figure can be found in Ref. [13].

The $+(-)$ sign refers to bound(virtual) state.

The behavior shown in Fig. 4 is reproduced for other core masses and energy ratios $E_{\mathrm{nn}} / E_{3}$. A curious fact is that for the same energy $E_{3}$ the size of the three-body systems follows the sequence: Borromean $<$ Tango $<$ Samba $<$ All-Bound. This apparent contradiction - intuitively we could expect a system with all two-body subsystems bound smaller than a system with all of them unbound - is immediately solved if we consider that for the same $E_{3}$ a system with a weaker interaction should be closer in order to form a bound three-body system.

\section{OUTLOOK}

Although we can study the relations between observables considering several halo nuclei, we are not able to change the interaction between nucleons. However, this change can be made in ultracold atomic traps via Feshbach resonance. Using this mechanism we can tune the two-body interaction changing the magnetic field. Several articles, most of them in the atomic context, have been written recently showing that universality is a hot topic $[12,15]$.

Obviously, the next frontiers to be investigated are the four and five-body problems [16], but certainly the three-body problem is not yet closed [17].

\section{ACKNOWLEDGMENTS}

M.T.Y. thanks FAPESP, CNPq and the XXXII RTFNB organizing committee for support; and L. Tomio and R.S. Marques de Carvalho for the comments on the manuscript. 


\section{REFERENCES}

1. E. Braaten and H.-W. Hammer, Phys. Rep. 428, 259-390 (2006).

2. A.S. Jensen, K. Riisager, D.V. Fedorov and E. Garrido, Rev. Mod. Phys. 76, 215-261 (2004).

3. T. Bjerge, Nature 138, 400-401 (1936).

4. I. Tanihata et al., Phys. Rev. Lett. 55, 2676-2679(1985).

5. T. Kobayashi et al., Phys. Lett. B 232, 51-55 (1989).

6. V. Efimov, Phys. Lett. B 33, 563-564 (1970).

7. T. Kraemer et al., Nature 440, 315-318 (2006).

8. S.K. Adhikari et al., Phys. Rev. A 37, 3666-3673 (1988).

9. M.T. Yamashita, T. Frederico, A. Delfino and L. Tomio, Phys. Rev. A 66, 052702 (2002).

10. S.K. Adhikari, T. Frederico and I.D. Goldman, Phys. Rev. Lett. 74, 487-491 (1995).

11. L. Platter, H.-W. Hammer and U.-G. Meissner, Phys. Rev. A 70, 52101 (2004). M.T. Yamashita, L. Tomio, A. Delfino and T. Frederico, Europhys. Lett. 75, 555-561 (2006).

12. J. von Stecher, J.P. D'Incao and C.H. Greene, Nature Phys. 5, 417-421 (2009).

13. M.T. Yamashita, L. Tomio and T. Frederico Nucl. Phys. A 735, 40-54 (2004).

14. F. Robicheaux, Phys. Rev. A 60, 1706-1709 (1999).

15. V. Efimov, Nature Phys. 5, 533-534 (2009); M. Zaccanti et al., ibid. 5, 586-591 (2009); G. Barontini et al. Phys. Rev. Lett. 103, 043201 (2009); F. Ferlaino et al., ibid. 102, 140401 (2009).

16. M.T. Yamashita, D.V. Fedorov and A.S. Jensen, arXiv:0911.1722

17. M.T. Yamashita, T. Frederico and L. Tomio, Phys. Rev. Lett. 99, 269201 (2007); Phys. Lett. B 660, 339-344 (2008). 
Copyright of AIP Conference Proceedings is the property of American Institute of Physics and its content may not be copied or emailed to multiple sites or posted to a listserv without the copyright holder's express written permission. However, users may print, download, or email articles for individual use. 